\title{
Long-Term Global Climate Dynamics: A Hopf Bifurcation Causing Recurrent Ice Ages
}

\author{
ANDREI KOROBEINIKOV ${ }^{\dagger}$ \\ a.korobeinikov@math.auckland.ac.nz \\ Department of Mathematics, University of Auckland, Private Bag 92019, Auckland, \\ New Zealand
}

ALEX MCNABB

a.mcnabb@auckland.ac.nz Department of Mathematics, University of Auckland, Private Bag 92019, Auckland, New Zealand

\begin{abstract}
Rapid and dramatic changes in climate and glacial conditions have taken place during the last 2.5 million years of the earth's history. Huge ice sheets expanded and contracted periodically, at times covering large areas of North America and Europe. Global sea levels dropped and rose $100 \mathrm{~m}$ to $150 \mathrm{~m}$ in response to the growth and melting of glaciers, causing continental coast lines to move far into present sea areas and then retreated again. We will use a simple conceptual model to demonstrate that these climate and glacier fluctuations can be a consequence of a supercritical Hopf bifurcation in models of the "ocean-land-atmosphere" system.
\end{abstract}

Keywords: Hopf bifurcation, climate fluctuations, glacials

\section{Introduction}

Dramatic changes have taken place during the last 2.5 million years of the earth's climatic history. Very cold and comparatively warmer phases alternating in quick succession have caused large ice sheets to expand and contract. Continents at times extended far into present sea areas as sea levels periodically dropped and rose $100 \mathrm{~m}$ to $150 \mathrm{~m}$ in response to the growth and melting of glaciers. Continents and islands that today are separated by oceans were during these intermittent ice ages connected by land bridges, and large parts of present-day desert areas were covered with vegetation.

With additional information obtained during the last few decades, a more detailed interpretation of past events has been made possible [1]. The early and middle Eocene climate was very warm, and no glaciers existed even in the high-latitude polar regions. Available records from central Europe

$\dagger$ Requests for reprints should be sent to Andrei Korobeinikov, Department of Mathematics, University of Auckland, Private Bag 92019, Auckland, New Zealand. 
show a gradual cooling from about 40 million years ago to the present time. About 2.5 million years ago dramatic climate fluctuations started (see fig. 1). From then on, throughout the late Pliocene and the Pleistocene, mid-latitude glaciers existed providing a fluctuating ice coverage over significant areas of the earth's surface. The amplitudes of these fluctuations increased considerably 0.9 million years ago, when the largest mid-latitude ice sheets formed and left their imprints.

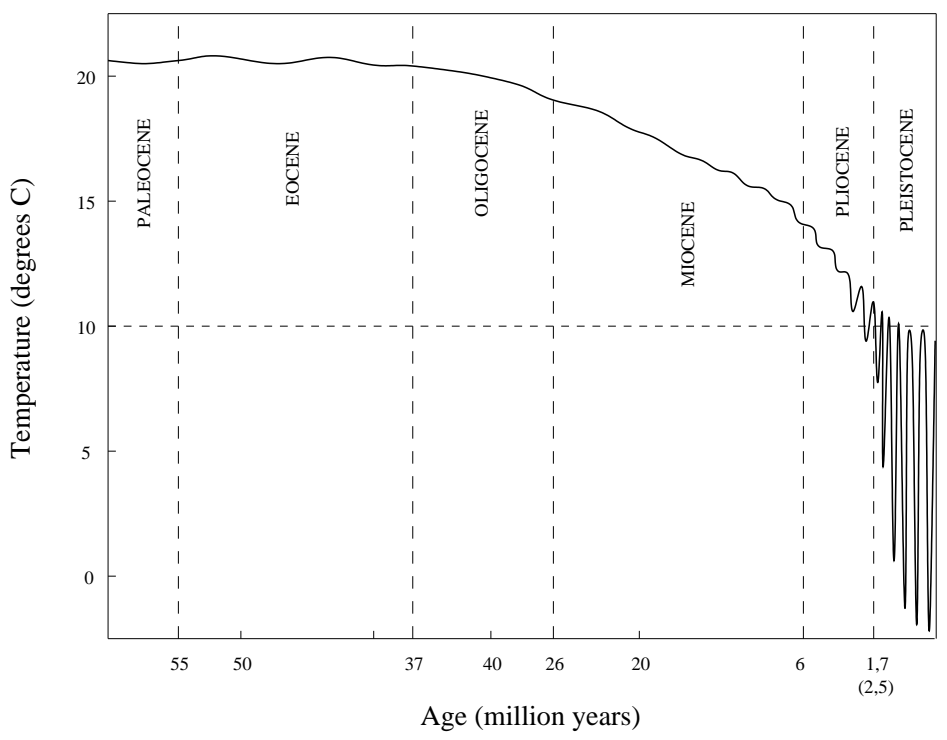

Figure 1. Mean annual temperature in central Europe for the last 50 million years (adapted from Andersen and Borns [1], fig. 1-19).

During the coldest phases, the true ice ages or glacials, ice sheets expanded over large areas of North America and Europe. Some of the coldest periods representing the glacial phases had mean annual temperatures in the order of $10-20^{\circ} \mathrm{C}$ lower than today in areas beyond the ice sheets in parts of Europe and USA. In parts of the subtropics and continental tropics temperatures were about $4-7^{\circ} \mathrm{C}$ lower, while along the marine equator, they were only slightly lower, or no lower, than today [1]. During the intermediate warmer phases, the interglacials, the climate and glacier conditions were about the same as they are today, although in the warmest parts of some interglacials the mean summer temperatures were in the order of $2^{\circ} \mathrm{C}$ warmer than today in much of Europe and North America. 
Indications for these glacier fluctuations are found both in oxygen-isotope and in terrestrial stratigraphy data [1].

Since the discovery of these climate and glacial fluctuations, scientists have speculated about their causes, and numerous theories have been proposed [1]. Some of the best known suggest the variations are a consequence of: 1 . astronomic factors; 2 . sun-spot activity; 3 . ocean currents; 4 . atmospheric composition; 5 . volcanic dust or dust from disintegrated meteorites in the atmosphere; 6 . surges of the Antarctic Ice Sheet; 7. rising or falling of parts of the earth's crust; 8. solar-terrestrial magnetic coupling; 9. fluctuation of the upper atmospheric jet-streams; 10 . asteroid impacts; 11 . interaction between ocean currents and atmospheric circulation; and 12. the "snowblitz" theory. (This recently formulated theory suggests a series of harsh winters and cool summers started the glaciations.) Objections have been presented against all of these theories, and many of them have been rejected outright as being too unlikely. A requirement that most of them fail to fulfil is an explanation of the fairly regular pattern of glacials and interglacials, as shown by the oxygen-isotope data [1].

A theory that seems to fit this pattern best is the astronomic Milankovitch theory, which attributes the climate and glacier fluctuations to eccentricity of the earth's orbit (which varies in 100000 years cycle), the obliquity (which varies between about $24.5^{\circ}$ and $22.5^{\circ}$ with 41000 years period) and the precession (which varies with $23000 / 19000$ years period). However, some serious objections have also been raised against this theory [1]. First of all, the theory requires glacial and climate fluctuations in the northern hemisphere to be out-of-phase with the fluctuations in the southern hemisphere whereas most field observations indicate that in fact the fluctuations are in phase in both hemispheres. Secondly, the calculated changes in energy-input to the earth's atmosphere and surface due to the Milankovitch-predicted changes are too minor when compared with the magnitude of the actual changes. And thirdly (and of most importance), according to this theory climate oscillations should have occurred throughout all of earth's history, whereas in fact the global climate seems to have been quite stable throughout most of it [1].

Presently available data [1] indicates the possibility for at least two different global climate regimes on earth. The first of them, observed for most of the earth's history, is a quite stable regime similar to that of the early and middle Eocene. The second, that which has prevailed throughout the late Pliocene and the Pleistocene, is an unstable regime whose distinctive feature is a succession of alternating warm and cold phases. Figure 1 demonstrates how the global climate shifts from one regime to the other. 
Our aim in this paper is to demonstrate that these climate and glaciers oscillations can be associated with a supercritical Hopf bifurcation in the global climate system. The term supercritical Hopf bifurcation refers to the shifting stability of an equilibrium leading to the development of a periodic orbit (a stable limit cycle) from a stable equilibrium point, as a parameter crosses a critical value. It implies rising stable oscillations of increasing amplitude in a system, once in a stable equilibrium state. Moreover, minor variations of a parameter's value can lead to dramatic change in the behaviour of such system. Qualitative descriptions of the climate and glacier dynamics during the Pliocene and the Pleistocene, and especially the fairly regular pattern of glacials and interglacials with their increasing in time amplitudes fit quite well the general pattern of behaviour of systems subject to a supercritical Hopf bifurcation.

\section{Model}

Water is by far the most abundant and important substance at the earth's surface. There are 1.4 billion $\mathrm{km}^{3}$ of it in its three phases: liquid water, ice, and water vapour. At present the earth's water is distributed as shown in the table 1.

Of course, the water distribution has varied during the Pleistocene. For instance, during the last glacial maximum 18,000 years ago, sea level dropped about $130 \mathrm{~m}$, accounting for a transfer of about $47 \times 10^{6} \mathrm{~km}^{3}$ of water (equal to about $3.5 \%$ of the oceanic water volume) from the oceans onto the land in form of ice and snow [3], while during interglacials practically all of the continents were ice-free.

We assume that long-term global water stocks are divided between two large interacting reservoirs labelled here as $O$ (for ocean) and $L$ (for land) (see fig. 2). The reservoir $O$ contains all ocean water of total mass $u$, including the ice of the ocean ice sheets. The reservoir $L$ contains water of total mass $v$ stored on land as snow and ice. Thus these reservoirs combined contain about $99.3 \%$ of the earth's surface water (see table 1 ).

Both reservoirs interact with the earth's atmosphere. We neglect the atmospheric water vapour (and thermal energy) content as small compared with the volumes of the oceans and the continental ice and snow.

We assume that these two reservoirs continuously exchange water mass and heat, and that there is a long term mean thermal energy content for each of them. The latter assumption is quite justified for long terms, since the thermal equilibrium in the systems ocean - air above and land - air above can be achieved comparatively quickly. (By long term we mean averaged over decades but varying slowly from one millennium to the next.) 
Table 1. Distribution of water on the earth's surface (adapted from Berner and Berner [2], p. 13).

\begin{tabular}{lcc}
\hline Reservoir & Volume $\left(10^{6} \mathrm{~km}^{3}\right)$ & Percent of Total \\
\hline Oceans & 1370 & 97.25 \\
Continental ice sheets & 29 & 2.05 \\
$\quad$ and glaciers & & \\
Groundwater & 9.5 & 0.68 \\
Lakes & 0.125 & 0.01 \\
Soil moisture & 0.065 & 0.005 \\
Atmosphere & 0.013 & 0.001 \\
Rivers & 0.0017 & 0.0001 \\
Biosphere & 0.0006 & 0.00004 \\
\hline \multicolumn{1}{c}{ Total } & 1408.7 & 100 \\
\hline
\end{tabular}

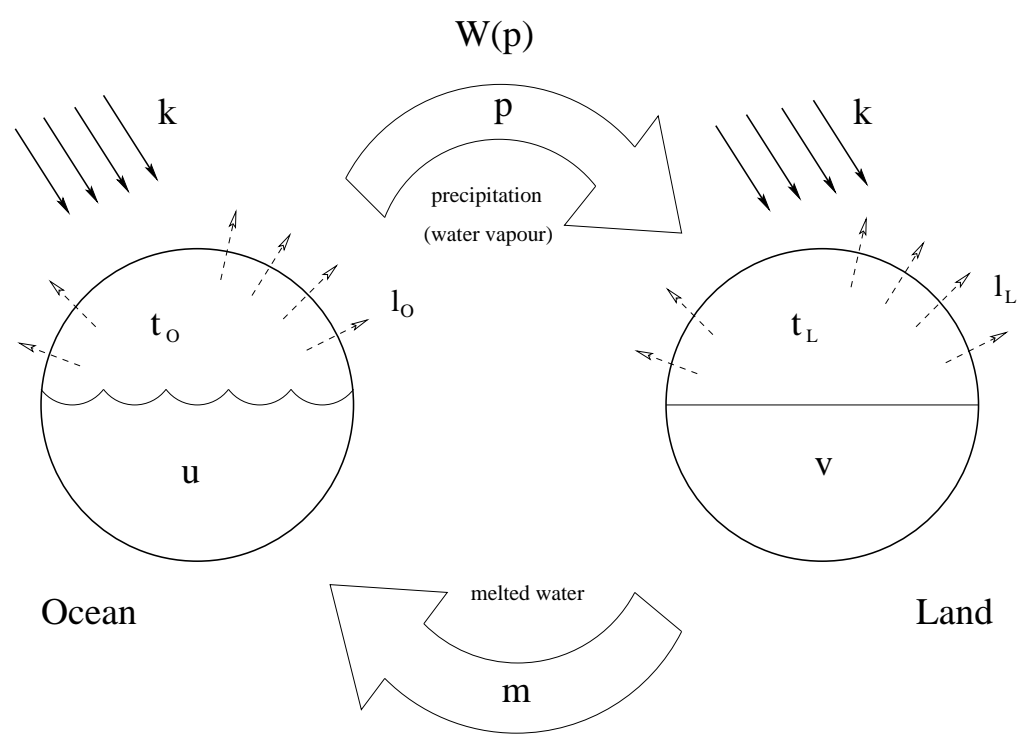

Figure 2. Global water balance. 
The global water mass $V$ at the earth's surface has remained fairly constant over geological time [2], p. 19, so that

$$
u+v=V=\text { constant }
$$

Variation of the water distribution during the Pleistocene is due to a continuous mass exchange between the oceans and the continental glaciers. Evaporated ocean water is transported onto the land as precipitation (rain, hail or snow). Snowfall tends to increase the mass of the continental ice sheets, while the rain-water and glacial melts quickly run off into the oceans through the continental river systems. Hence the function $v(t)$ satisfies

$$
\dot{v}=s-m
$$

where $s(t)$ is the rate of snowfall on the land, $m(t)$ is the rate at which the ice and snow reserves melt.

The primary energy source for the earth's surface is solar radiation. It forms $99.98 \%$ of the total energy flux to the earth's surface. (Geothermal heat provides some $0.018 \%$ and tidal energy adds another $0.002 \%$ of the total energy flux [2], p. 20 .) Consequently, solar radiation is the dominant influence on circulation of the atmosphere and oceans and the only energy source that will be considered in this paper.

Both reservoirs are continuously absorbing incoming short-wave (from 0.3 to $5 \mu \mathrm{m}$ [5], p. 26) solar radiation of intensity $k$ at rates $E_{O}$ and $E_{L}$, converting it to stored thermal energy. Both reservoirs are also continuously emitting energy, re-radiating it at rates $l_{O}$ and $l_{L}$ as longer-wave (from 4 to $100 \mu \mathrm{m}[5]$, p. 26) infrared earth's radiation into open space. We assume the heat exchange between the reservoirs is mainly via latent heat transfer. Latent heat is injected into the atmosphere above the oceans as water vapour. There it could be transported onto the continents as precipitation, and subsequently released by condensation (and even further by freezing in the case of snowfall) into the atmosphere. The heat must be also spent to melt the continental ice or snow. Hence the heat fluxes to and from the reservoirs $O$ and $L$ satisfy the equations

$$
\begin{aligned}
& \dot{q}_{O}=E_{O}-\gamma_{v} p-W(p)-l_{O}, \\
& \dot{q}_{L}=E_{L}+\gamma_{v} p+\gamma_{m} s-\gamma_{m} m-l_{L},
\end{aligned}
$$

where $q_{O}(t), q_{L}(t)$ are the heat contents of the reservoirs $O$ and $L ; p(t)$ is the total precipitation (snowfall $s(t)$ plus rainfall $r(t))$ rate; $\gamma_{v}, \gamma_{m}$ are the latent heats of vaporisation and melting for water; $W(p)$ is the heat spent on work to transport the water of mass $p$ from the ocean onto the land. 
The system (2), (3), (4) is in equilibrium when

$$
s=m, \quad E_{O}=W(p)+l_{O}+\gamma_{v} p, \quad E_{L}=l_{L}-\gamma_{v} p .
$$

For the purpose of further discussion we make some conjectures and assumptions that allow us to define the functions of the system (2), (3), (4) and the relationships governing the variables.

Assume that the total flux of water from the oceans onto the land (the total precipitation rate $p$ ) is proportional to the difference between the mean ocean temperature $t_{O}$ and the mean land temperature $t_{L}$, i.e.

$$
p=s+r=a\left(t_{O}-t_{L}\right) .
$$

The total precipitation is composed of rain and snow with a constant ratio of the snow $s(t)$ to the total precipitation $p(t)$, i.e.

$$
s=\delta p, \quad \text { where } \delta \cong 0.5 .
$$

We neglect the work $W(p)$ spent on the transportation of the precipitation, i. e.

$$
W(p)=0,
$$

since a comparatively small part (about $0.7 \%$ [2], p. 27) of the incoming solar radiation is converted into the energy of motion of ocean currents, winds, and waves.

The rate of snow melting $m(t)$ depends on the temperature of the atmosphere above the land $t_{L}$ and (since ice and snow melts on its surface) on the size of the ice surface area rather than the ice mass. Hence

$$
m=b t_{L} v^{\kappa}, \quad \text { where } \quad 0<\kappa<1 .
$$

The rates of heat emission $l_{O}, l_{L}$ are directly proportional to the temperature in the reservoirs

$$
l_{O}=c t_{O}, \quad l_{L}=c t_{L}
$$

We assume that the rate of absorption of solar radiation by the ocean is proportional to the solar radiation intensity at the earth's surface $k$ only, i. e.

$$
E_{O}=k \alpha
$$

Of course, the rate of the solar radiation absorption by the ocean depends on the ocean's surface area. However, since the continental shelf in the tropics is narrow, the tropical area of the oceans remains fairly constant as the ocean's level varies from $100 \mathrm{~m}$ to $150 \mathrm{~m}$. 
The rate of the absorption of solar radiation by the land is proportional to the solar radiation intensity $k$, and (since white snow reflects more than $70 \%$ of the incoming solar radiation in contrast to $10-20 \%$ from some forests) depends inversely on the ice and snow surface area. We define it by the expression

$$
E_{L}=k \beta\left(1-\frac{v^{\kappa}}{M}\right), \quad \text { where } \quad M>0 .
$$

The mean temperatures dependence of both reservoirs is assumed to be given by the expressions

$$
C_{O} t_{O}=q_{O}, \quad C_{L} t_{L}=q_{L}
$$

where $C_{O}$ is the total mean heat capacity of the oceans water and $C_{L}$ is the total mean heat capacity of the reservoir $L$. The heat $q_{L}$ is stored mainly by the atmosphere, and $C_{L}$ is the total heat capacity of the atmosphere in the reservoir $L$ at the constant volume.

Under the assumptions (5)-(12) the equations (2), (3), (4) take the form

$$
\begin{aligned}
\dot{v} & =a \delta t_{O}-a \delta t_{L}-b t_{L} v^{\kappa} \\
\dot{t}_{O} & =k \frac{\alpha}{C_{O}}+\frac{\gamma_{v} a}{C_{O}} t_{L}-\frac{\left(\gamma_{v} a+c\right)}{C_{O}} t_{O}, \\
\dot{t}_{L} & =k \frac{\beta}{C_{L}}-k \frac{\beta}{M C_{L}} v^{\kappa}+\frac{\gamma a}{C_{L}} t_{O}-\frac{\gamma a+c}{C_{L}} t_{L}+\frac{b \gamma_{m}}{C_{L}} t_{L} v^{\kappa},
\end{aligned}
$$

where $\gamma=\gamma_{v}+\delta \gamma_{m}$. The condition

$$
B^{2}-4 A C>0
$$

where

$$
A=c\left(c+2 a \gamma_{v}\right), \quad B=\alpha a \gamma_{v}+\beta\left(a \gamma_{v}+c\right)+c \sigma, \quad C=\alpha \sigma, \quad \sigma=\frac{a \beta \delta}{M b},
$$

ensures that the system (13), (14), (15) has two equilibria $Q_{1}$ and $Q_{2}$ given by

$$
\bar{v}=\left[\frac{a \delta\left(\alpha-c t^{*}\right)}{b t^{*}\left(c+a \gamma_{v}\right)}\right]^{\frac{1}{\kappa}}, \quad \bar{t}_{O}=\frac{\alpha+a \gamma_{v} t^{*}}{c+a \gamma_{v}} k, \quad \bar{t}_{L}=t^{*} k
$$

Here

$$
\left(t^{*}\right)_{i}=\frac{B \pm \sqrt{B^{2}-4 A C}}{2 A} \quad(i=1,2) .
$$

(The sign "+" for $Q_{1}$ and "-" for $Q_{2}$.) It is easy to see that $t^{*}$ and $\bar{v}$ do not depend on $k$ while $\bar{t}_{O}$ and $\bar{t}_{L}$ depend on $k$ linearly. 
It is reasonable to demand that both of the equilibria $Q_{1}, Q_{2}$ be located in the positive area of the phase space. If the equilibria $Q_{1}, Q_{2}$ exist, then both $\bar{t}_{O}, \bar{t}_{L}$ are positive. The condition

$$
\alpha-c t^{*}>0
$$

provides that $\bar{v}>0$ holds for both equilibria. If conditions (16) and

$$
2 \alpha c+4 \alpha a \gamma_{v}-B>0
$$

hold, then (18) is equivalent to the condition

$$
\begin{aligned}
&\left(2 \alpha c+4 \alpha a \gamma_{v}-B\right)^{2}-B^{2}+4 A C \\
&= {\left[2 \alpha c+3 \alpha a \gamma_{v}-\beta\left(a \gamma_{v}+c\right)-c \sigma\right]^{2} } \\
& \quad-\left[a \alpha \gamma_{v}+\beta\left(a \gamma_{v}+c\right)+c \sigma\right]^{2}+4 c \alpha \sigma\left(c+2 a \gamma_{v}\right) \\
&= 4 \alpha\left(c+2 a \gamma_{v}\right)\left[\alpha\left(a \gamma_{v}+c\right)-\beta\left(a \gamma_{v}+c\right)-c \sigma\right]+4 \alpha\left(c+2 a \gamma_{v}\right) c \sigma \\
&= 4 \alpha(\alpha-\beta)\left(c+2 a \gamma_{v}\right)\left(c+a \gamma_{v}\right)>0,
\end{aligned}
$$

which holds provided

$$
\alpha>\beta \text {. }
$$

We assume for further discussion that both conditions (16) and (19) hold. These conditions seem to be realistic.

\section{Supercritical Hopf bifurcation}

A basic feature of the system (13), (14), (15) is it has possibilities for the occurrence of a supercritical Hopf bifurcation.

THEOREM 1 If

$$
\bar{\eta}=\frac{b\left(\gamma_{v} a+c\right)\left(C_{O}+C_{L}\right) t^{*}}{C_{O}}-\frac{b \sigma}{a \gamma_{v}+c}\left(\frac{\alpha}{t^{*}}+a \gamma_{v}\right)<0,
$$

then the system (13), (14), (15) with the conditions (16), (19) admits a supercritical Hopf bifurcation of the equilibrium $Q_{1}$ to a stable closed orbit, with the solar radiation intensity $k$ as the bifurcation parameter. If $\bar{\eta} \geq 0$, then the equilibrium $Q_{1}$ is stable for all $k>0$.

The equilibrium $Q_{2}$ is unstable for all $k>0$.

Proof: According to the Hopf bifurcation theorem [6], p. 18-25 a supercritical Hopf bifurcation occurs when a pair of isolated nonzero, simple complex conjugate eigenvalues $\lambda(k), \overline{\lambda(k)}$ of the linearised system crosses 
the imaginary axis from left to right at $k=k_{c r}$ at nonzero speed, i.e., $\operatorname{Re} \lambda\left(k_{c r}\right)=0$ and $\left.\frac{d(\operatorname{Re} \lambda(k))}{d k}\right|_{k=k_{c r}}>0$, while the rest of the spectrum remains in the open left-half plane.

Linearization of the system (13), (14), (15) in the neighbourhood of the equilibrium $Q_{i}=\left(\bar{v}, \bar{t}_{O}, \bar{t}_{L}\right)_{i}(i=1,2)$ gives

$$
\begin{aligned}
\dot{v} & =-\kappa b \bar{t}_{L} \bar{v}^{\kappa-1} v+a \delta t_{O}-\left(a \delta+b \bar{v}^{\kappa}\right) t_{L}, \\
\dot{t}_{O} & =-\frac{\gamma_{v} a+c}{C_{O}} t_{O}+\frac{\gamma_{v} a}{C_{O}} t_{L}, \\
\dot{t}_{L} & =-\frac{\kappa \bar{v}^{\kappa-1}}{C_{L}}\left(\frac{k \beta}{M}+b \gamma_{m} \bar{t}_{L}\right) v+\frac{a}{C_{L}} \gamma t_{O}-\frac{a \gamma+c+b \gamma_{m} \bar{v}^{\kappa}}{C_{L}} t_{L} .
\end{aligned}
$$

The characteristic equation of the linearised system (20), (21), (22) is

$$
\lambda^{3}+a_{1} \lambda^{2}+a_{2} \lambda+a_{3}=0
$$

where

$$
a_{1}=\eta_{1} k+\xi_{1}, \quad a_{2}=\eta_{2} k+\xi_{2}, \quad a_{3}=\eta_{3} k,
$$

and

$$
\begin{array}{rlrl}
\eta_{1} & =\kappa b t^{*} \bar{v}^{\kappa-1}, & \xi_{1} & =\frac{\gamma_{v} a+c}{C_{O}}+\frac{\gamma a+\gamma_{m} b \bar{v}^{\kappa}+c}{C_{L}}, \\
\eta_{2} & =\frac{\kappa \bar{v}^{\kappa-1}}{C_{L}} \bar{\eta}, & \xi_{2} & =\frac{\left(a \gamma_{v}+c\right)\left(c+b \gamma_{m} \bar{v}^{\kappa}\right)+a c \gamma}{C_{O} C_{L}}, \\
\eta_{3} & =\frac{\kappa \bar{v}^{\kappa-1}}{C_{O} C_{L}}\left[b c\left(c+2 a \gamma_{v}\right) t^{*}-\frac{b \alpha \sigma}{t^{*}}\right]= \pm \frac{\kappa \bar{v}^{\kappa-1} b}{C_{O} C_{L}} \sqrt{B^{2}-4 A C},
\end{array}
$$

"+" refers to $Q_{1}$ and "-" to $Q_{2}$.

Since $\eta_{3}<0$ and, consequently, $a_{3}=\eta_{3} k=-\lambda_{1} \lambda_{2} \lambda_{3}<0$ for the equilibrium $Q_{2}$, then for all $k>0$ either one of the eigenvalues, or all three of them have positive real part. Consequently, the equilibrium $Q_{2}$ is unstable for all $k>0$. We will further consider only the equilibrium $Q_{1}$.

For all positive parameters of the system (13), (14), (15) in the equilibrium $Q_{1}$ the inequalities $\eta_{1}, \eta_{3}, \xi_{1}, \xi_{2}>0$ and $a_{1}, a_{3}>0$ hold. The coefficient $\eta_{2}$ depends on the sign of $\bar{\eta}$ and could be either positive or negative. However, even if $\eta_{2}<0$, we still have $a_{2}>0$ for $0<k<\frac{\xi_{2}}{\left|\eta_{2}\right|}$.

The conditions of the Hopf bifurcation theorem are satisfied if $a_{i}>0(i=$ $1,2,3)$ and $\Delta=a_{1} a_{2}-a_{3}=0$. The first conditions, $a_{i}>0$, are just the Routh-Hurwitz conditions for all roots of the characteristic equation (23) to be in the open left-half plane, and the second property, $\Delta=a_{1} a_{2}-a_{3}=$ 0 ,together with $a_{i}>0$ are sufficient conditions for the existence of one 
negative real root and a pair of pure imaginary roots [4], p. 197. The second condition

$$
\Delta=a_{1} a_{2}-a_{3}=d_{1} k^{2}-d_{2} k+d_{3}=0
$$

is satisfied at $k=k_{1}$ and $k=k_{2}$ given by

$$
k_{1}=\frac{d_{2}-\sqrt{d_{2}^{2}-4 d_{1} d_{3}}}{2 d_{1}}, \quad k_{2}=\frac{d_{2}+\sqrt{d_{2}^{2}-4 d_{1} d_{3}}}{2 d_{1}} .
$$

Here

$$
d_{1}=\eta_{1} \eta_{2}, \quad d_{3}=\xi_{1} \xi_{2}
$$

and

$$
\begin{aligned}
d_{2} & =\eta_{3}-\xi_{1} \eta_{2}-\xi_{2} \eta_{1} \\
& =-\frac{b \kappa \bar{v}^{\kappa-1}}{C_{O} C_{L}}\left(\frac{C}{t^{*}}+\gamma_{m}\left(\left(a \gamma_{v}+c\right) b \bar{v}^{\kappa}+a c \delta\right) t^{*}\right)-\xi_{1} \eta_{2}
\end{aligned}
$$

Since $\eta_{1}>0$ always, the sign of $d_{1}$ depends on the sign of $\eta_{2}$ (which can be either negative or positive); and $d_{3}>0$ always since $\xi_{1}, \xi_{2}>0$.

A supercritical Hopf bifurcation occurs when two complex conjoint eigenvalues cross the imaginary axis from left to right, i.e. when $\Delta=a_{1} a_{2}-a_{3}$ change sign from positive to negative. Depending on the sign of $\bar{\eta}$ (which can be either negative or positive), there are three possibilities.

1. If $\bar{\eta}>0$, then $\eta_{2}>0$, and hence $d_{1}=\eta_{1} \eta_{2}>0$ while $d_{2}<0$. In this case both the roots $k_{1}, k_{2}$ of the equation (24) are either real negative, or complex with negative real part. For all $k>0$ the Hurwitz determinant $\Delta$ is positive and $a_{i}>0(i=1,2,3)$. Hence for all $k>0$ all roots of the characteristic equation (23) are located in open left-half of the complex plane. Consequently, the equilibrium $Q_{1}$ is always stable, and no supercritical Hopf bifurcation is possible in this case.

2. If $\bar{\eta}=0$, then $\eta_{2}=0$ and $d_{1}=0$ too. Consequently, the equation (24) has the only root $k_{0}$ given by

$$
k_{0}=\frac{d_{3}}{d_{2}}=\frac{\xi_{1} \xi_{2}}{\eta_{3}-\xi_{2} \eta_{1}} .
$$

In this case

$$
d_{2}=\eta_{3}-\xi_{2} \eta_{1}=-\frac{b \kappa \bar{v}^{\kappa-1}}{C_{O} C_{L}}\left(\frac{C}{t^{*}}+\gamma_{m}\left(\left(a \gamma_{v}+c\right) b \bar{v}^{\kappa}+a c \delta\right) t^{*}\right)<0,
$$


hence $k_{0}<0$ too. For all $k>0$ the Hurwitz determinant $\Delta$ is positive and $a_{i}>0(i=1,2,3)$. Hence for all $k>0$ all roots of the characteristic equation (23) are located in open left-half of the complex plane. Consequently, the equilibrium $Q_{1}$ is always stable, and no supercritical Hopf bifurcation is possible in this case as well.

3. If $\bar{\eta}<0$, then $\eta_{2}<0$, hence $d_{1}<0$ and $d_{3}>0$. In this case the equation (24) always has two real roots $k_{1}, k_{2}$ given by (25). Furthermore, $k_{1}$ is always positive while $k_{2}<k_{1}$ is always negative. In this case for $k>0$ the equilibrium $Q_{1}$ is stable in the interval $0<k<k_{1}$. At $k_{c r}=k_{1}$ the Hurwitz determinant $\Delta$ changes sign from positive to negative, and a supercritical Hopf bifurcation occurs. Since $\Delta=a_{1} a_{2}-a_{3}=0$ at $k=k_{1}$, and $a_{1}, a_{3}$ are always positive, then $a_{2}=a_{3} / a_{1}>0$ too at point $k=k_{1}$. Hence the inequality $0<k_{1}<\xi_{2} /\left|\eta_{2}\right|$ holds.

This completes the proof.

\section{Discussion}

According to the theorem, the bifurcation is triggered by an increase of the solar radiation intensity. This fact might appear to be surprising. However, this was expected and predicted by the authors. In an ice age an enormous quantity of ice was deposited in the continental glacials. To evaporate and transport these volumes of water onto the continents the global water pumping machine must be provided with some excess of energy, and the Sun is the primary energy source for the earth's surface. This explains also why the solar radiation intensity $k$ have been chosen as a bifurcation parameter.

We should note that the increase of $k$ does not necessarily imply the rise of the Sun's activity. In fact, the matter is not how much the Sun radiates but how much reaches the earth's surface. A number of factors can lead to the increase of parameter $k$ value. Thus, apart from variation of the Sun's activity and the earth's position corresponding to the Sun, the rise of the solar radiation intensity on the earth's surface can be a consequences of the earth's atmosphere evolution. Since we have no evidence that the Sun's activity or the earth's position varied considerably, the authors are inclined to believe the last possibility.

It is notable that at present only about $55 \%$ of incoming solar radiation reaches the earth's surface [2], p. 21. The rest, $45 \%$ (!), is absorbed or reflected by the atmosphere. It can be assumed (for example, the greenhouse effect theory) that the variation of the atmosphere's transparency (due to 
the composition or the physical properties) can cause the variation of these figures and consequently, the variation of the parameter $k$ value.

In the case of the suggested model, the variation of the earth's atmosphere transparency (which depends on the composition, density or other physical properties) can affect the system behaviour in two different ways. First, it can decrease or increase the portion of the incoming solar radiation which reaches the earth's surface, and in consequence cause the Hopf bifurcation. Second, it can increase or decrease the portion of the longer-wave earth's radiation which leaves the earth's atmosphere. Though it does not follow directly from our analysis, this can stabilise or destabilise the system as well.

The above-considered model comprises two factors, each of which might destabilise the global climate. Firstly, the absorption of the solar radiation by land is inversely dependent on the area of ice-coverage. Secondly, the ice and snow melts from its surface and not in its volume. A third factor - the possible inverse dependence of the portion of snow in the total precipitation - though indicated, was not included in the model for the sake of simplicity and manageability.

Apart of the parameter $k$, the model combines twelve other parameters. Variation of some of these other parameters could (technically at least) trigger the bifurcation. However, it is extremely unlikely that these other parameters had any significant variation for say last 20 million years.

\section{Conclusion}

The prime aim of this paper was to demonstrate that a Hopf bifurcation in a model of the global climate system could (or rather must) cause dramatic changes in the global climate similar to those observed throughout the Pliocene and the Pleistocene. We would like to stress again that available records demonstrate that throughout geological history the global climate has exhibited two very different types of behaviour. For most of geological history the global climate seems to have been quite stable. However, during more recent times, climate stability had shifted giving rise to climate behaviour which could be described as "unstable" and "chaotic", and whose distinctive feature is a recurrent succession of the very cold phases altering with comparatively warm periods. Such behaviour, connected with changing stability and the development of violent oscillations, fits the general pattern of supercritical Hopf bifurcations.

In the multi-variable systems the events could get a further development. While a single bifurcation leads to the birth of a stable limit cycle (responsible for self-oscillations in a system), possible further bifurcations in 
a high-dimensional dynamical system might lead to very complicated behaviour usually described as "chaotic".

The actual mechanisms, which govern the earth's climate, still remain for the most part a mystery. The specific interactions between parts of the hyper-complex global mechanical and thermodynamical "ocean-atmosphereland" system are hard to determine as the available evidence is far too deficient. Under the circumstances, simple conceptual qualitative modeling is a start towards understanding its nature. We do not pretend that the extremely simple model suggested above in this paper is able to explain adequately all aspects of climate dynamics. Furthermore, we deliberately limited our presentation to a simple, even naive, model in order to demonstrate qualitative (and only qualitative) features of global ocean-atmosphere-land interactions, and how these interactions could lead to climate behaviour known from field data.

In conclusion we would like to add that we have considered only one destabilising mechanism here. In reality many others destabilising mechanisms are possible. For instance, the role of atmospheric carbon dioxide is not yet clear, though it is known that its contents in the atmosphere correlates with temperature [1], p. 28.

\section{References}

1. B. G. Andersen and H. W. Borns Jr. The Ice Age World. Scandinavian University Press, Oslo, 1994.

2. E. K. Berner and R. A. Berner. The Global Water Cycle. Prentice-Hall, London, 1987.

3. A. L. Bloom. Glacial-eustatic and isostatic controls of sea level since the last glaciation. In The late Cenozoic ice age, (Edited by K.K. Turekian), pp. 355-379. Yale Univ. Press, New Haven, 1971.

4. F. R. Gantmacher. The Theory of Matrices, v. 2. Chelsea Pub. Co., NewYork, 1959.

5. B. A. Kagan. Ocean-Atmosphere Interaction And Climate Modelling. Cambridge University Press, Cambridge, 1995.

6. J. E. Marsden and M. McCracken. The Hopf bifurcation and its applications. Springer, New York, 1976. 


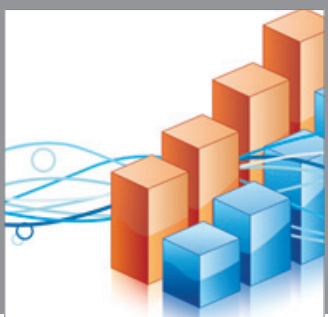

Advances in

Operations Research

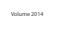

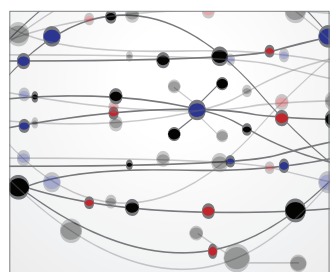

\section{The Scientific} World Journal
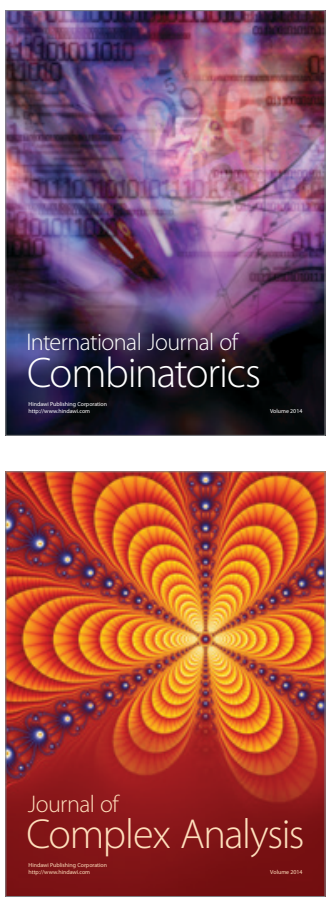

International Journal of

Mathematics and

Mathematical

Sciences
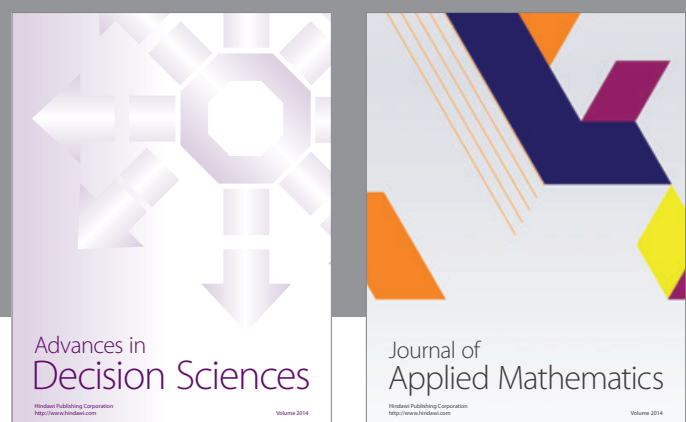

Journal of

Applied Mathematics
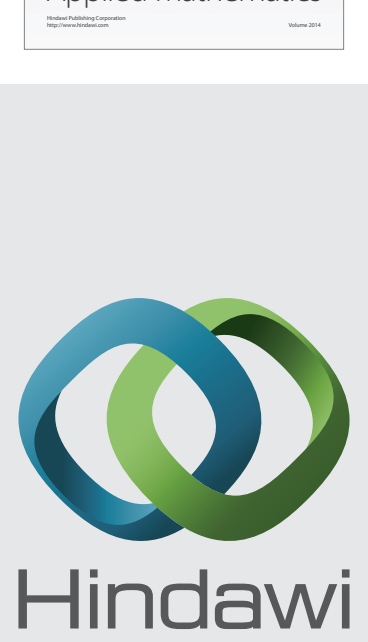

Submit your manuscripts at http://www.hindawi.com
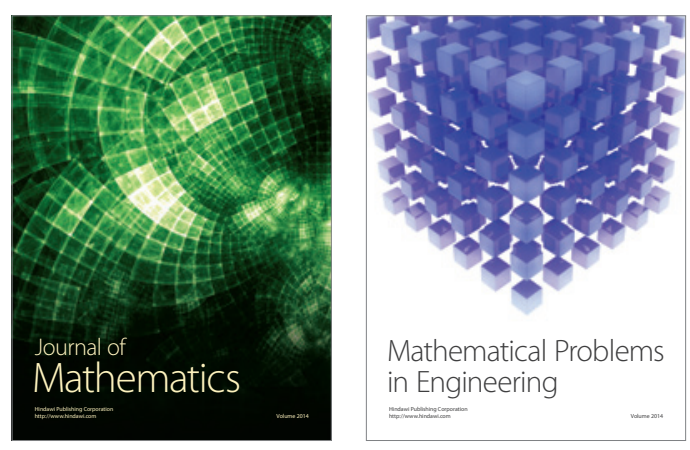

Mathematical Problems in Engineering
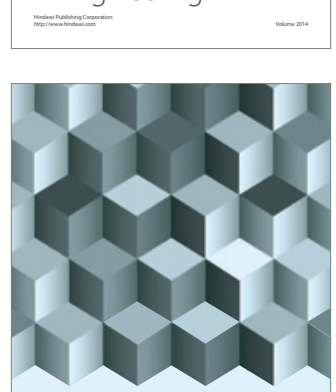

Journal of

Function Spaces
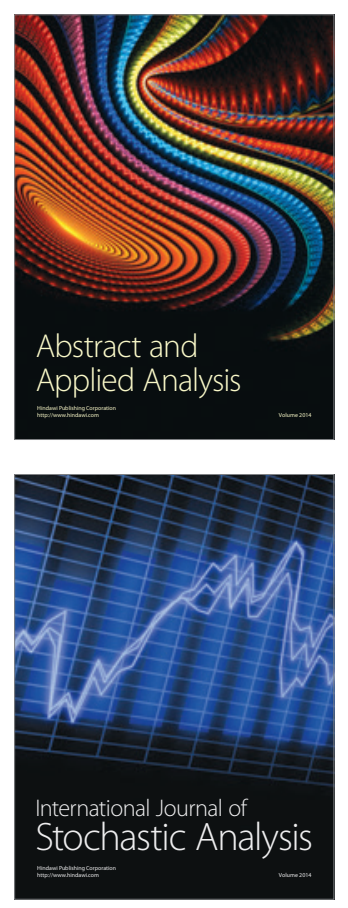

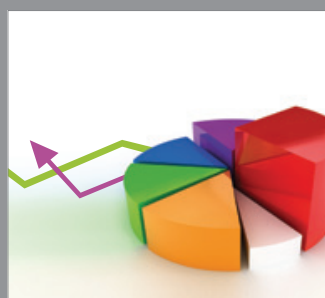

ournal of

Probability and Statistics

Promensencen
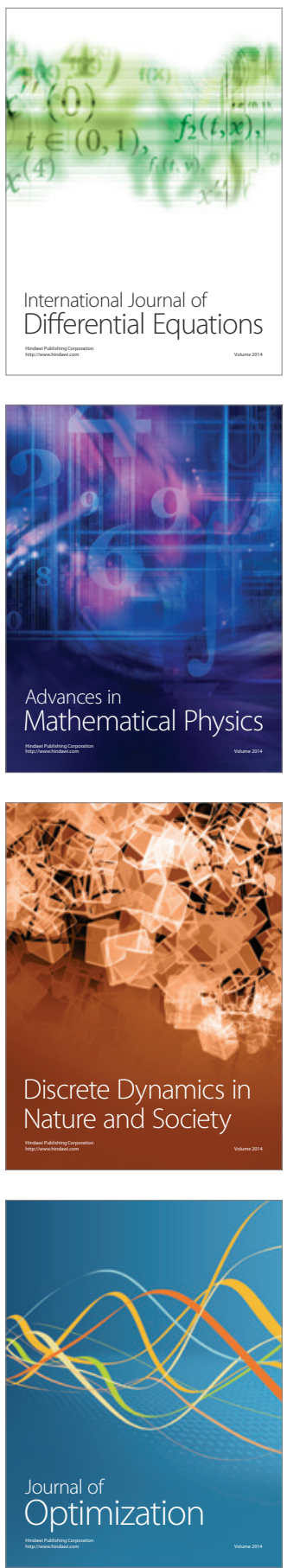